$\xi=-1$

\title{
An Optimal Panoramic Strategy for Women safety using IoT
}

\author{
Afiya Parveen Begum ${ }^{1 *}$, Dr. S. Satyanarayana ${ }^{2}$, Dr. K. Bhagavan ${ }^{3}$ \\ ${ }^{1}$ M.Tech Scholar, Department of Computer Science, Koneru Lakshmaiah Educational Foundation, Guntur, Andhra Pradesh, India \\ ${ }^{2}$ Professor, Department of Computer Science, Koneru Lakshmaiah Educational Foundation, Guntur, Andhra Pradesh, India \\ ${ }^{3}$ Professor, Department of Computer Science, Koneru Lakshmaiah Educational Foundation, Guntur, Andhra Pradesh, India \\ *E-mail: afiyaparveen786@gmail.com
}

\begin{abstract}
This paper provides you an idea about Internet of Things and reviews the origin of IoT, key enabling technologies, IoT architecture, IoT in industrial areas, applications of IoT, Operating system used in IoT devices. As the Internet of Things(IoT) at large will foster billions of devices, people and services to interconnect and exchange information and useful data. We have proposed a real-time application device of IoT useful for women safety in today's world. Considering the issues increasing on women atrocities, the Universal key question raising in every girl's mind is when they will have a freedom to walk freely in the society even in odd hours without worrying about their security. This paper will help you to use technology with a new perspective for women safety and to provide proof-based evidence against the perpetrators of crime against women. Every Day women around the world are Harassed, Raped, and Killed. The change in society should come and leads to the time when media broadcast more of women successes rather than harassment. Since we can't respond pertinently in perilous situations, we need a device which automatically senses and saves the victim is one of the idea of this paper.
\end{abstract}

Keywords: Internet of Things; Architecture; women security; Raspberry Pi; GSM.

\section{Introduction}

IoT is presently not just about a Technology worldwide but a reality. It is the logical step in the evolution of the Internet. As we live in the AGE OF AFTER GOOGLE where information is just one touch and one click away. The IoT is near future of AG. The IoT technology is enormously developing day-by-day because of continues efforts of the wide community, stretching from hobbyists all the way to researches and getting more attentiveness due to the evolution of wireless technology. IoT is an Umbrella term for a wide range of underlying technologies and services such as analytics, Artificial Intelligence, Big Data etc. Initially, the term refers to uniquely recognizable interoperable connected objects with RFID technology [1], afterward many research correlate IoT with many technologies such as embedded sensing and communication possibility devices.

Today a commonly accepted definition is: IoT is a system of a dynamic global network infrastructure with self-configuring capabilities and interrelated computing devices, machines, objects, animals and people with unique IP address and use intelligent interfaces that are integrated into the network to transmitting the data without requiring person-to person or person-to-computer interaction. Specifically, the integration of sensor, actuators, RFID tags, and communication technology functions as the base of IoT and explain how a numerous heterogenous devices around us connect and communicate with one another to reach the common goal [2]. As IoT is evolving reality, unquestionably the strength is its visibility in both working and domestic facets of daily-life and behavior of potential users and similarly from the perspective of the business user. Real life IoT deployment across a variety of sectors such as IoT in business \& industries, fields of automation and industrial manufacturing, intelligent transportation, logistics etc and IoT in consumer space where there are many thousands of devices \& applications for a broad variety of purposes. According to research survey by 2020 , nearly 50 billion of IoT devices are connected to the Internet. As the technology is fast evolving, threats deriving from widespread technology are also stressed. Many challenging threats/issues should be addressed \& freed before the IoT being accepted. Currently, many standardization, researches \& industries are involved in the development of such solution IoT facing today in order to highlight the technological requirements.

The paper is summarized as 2. The Origin of IoT. 3.IoT enabling technologies. 4. IoT architecture. 5.Role of IoT in industries. 6.IoT Applications 7. Operating Systems used in IoT devices. 8.Existing system 9. Proposed System 10. conclusion.

\section{The Origin of IoT:}

RFID is an initial technology for IoT, which allows Microchips to transmit the identification data to the reader. It is root technology and an initial path for the development of IoT vision, but it is not the only one. RFID allows to identify, track, and monitor the objects combined with RFID tags automatically [3]. The most reallife implementation of RFID is logistic, retailing, supply chain management, warehouses, public transportation, identification, electronic toll collection, etc [4][5]. The growing usage of RFID decreases the cost of RFID and increases the standardization. Many technologies along with RFID are key components for full deployment of IoT. Internet of Things is coined by Kevin Ashton in a presentation to Proctor \& Gamble in 1999 [6] (P\&G), he is an Executive director and Co-founder of MIT'S Auto-ID center. It is the big year for IoT \& MIT. He came up with an idea of "When 
Things Start to Think". The Word IoT (Internet of Things) has many definitions which let the reader feel difficult in understanding what is IoT? and which basic idea stands beside it. The word IoT is buzzword realm on information technology create an apparent fuzziness, because it syntactically consists of 2 terms. The first "Internet" which focus on the network-oriented view of IoT, and another is on generic "Objects". In Fig 1 [9], concepts, technologies, and standards of IoT vision are highlighted. The term IoT broadly refers to both: (i) the global network communicating with smart objects by means of Internet Technologies. (ii) the set of supporting technologies necessary to realize such a vision including RFID's, sensor/actuators, Machine-to-Machine communication devices etc. (iii) the set of services and application leveraging such technologies to open new business and markets opportunities.

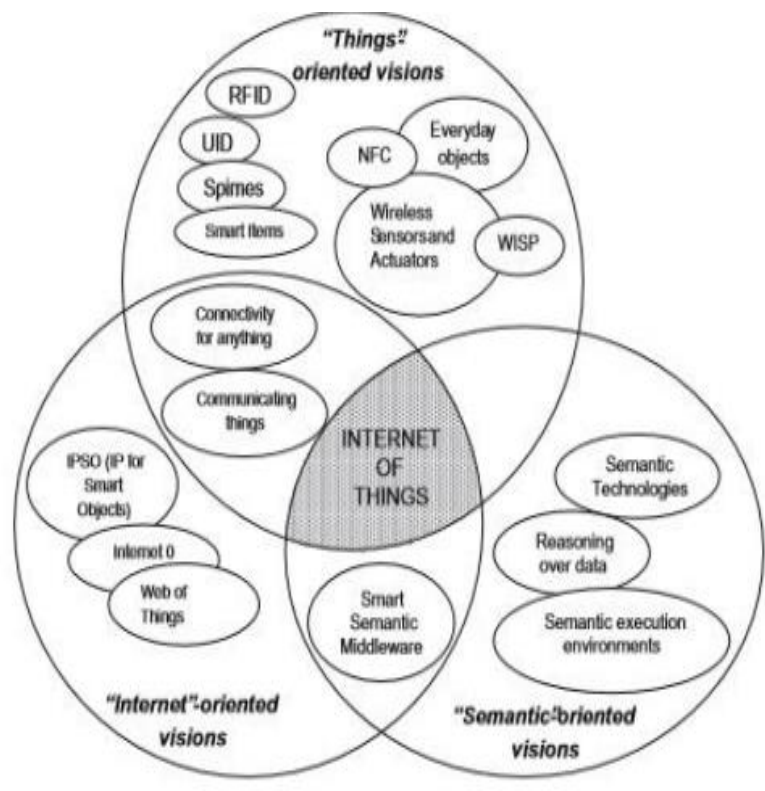

Fig 1: "Internet of Things" in different visions.

The MIT's Auto-ID labs [7], a world-Wide network of academics' research laboratories in the field of networked RFID and emerging sensing technologies. They're primarily focus on the development of the Electric Product Code TM (EPC) to support the spreading use of RFID in worldwide modern trading network. IoT is not just a global EPC [8] system in which the only objects are RFID's. Another groundwork technology for IoT is Wireless SN used to interconnect the sensors data to sense and monitoring its application such as environmental, healthcare, industrials [10] [11]. The RFID, WSN, and many other additional technologies are shown in Fig 2[18] significantly contribute to the development of IoT [12][17].

\begin{tabular}{|c|c|c|c|c|c|}
\hline RFID & Auto ID & ITU IoT & \multirow{2}{*}{\multicolumn{2}{|c|}{$\begin{array}{l}\text { RFID CMOS } \\
\text { Smartphone } \\
\end{array}$}} & \multirow{5}{*}{$\begin{array}{c}\text { Internet } \\
\text { of } \\
\text { Things }\end{array}$} \\
\hline & & Barcodes & & & \\
\hline & WSN & \multicolumn{3}{|c|}{ Could Computing } & \\
\hline & \multicolumn{3}{|c|}{ Location based service } & SoA & \\
\hline & & \multicolumn{3}{|c|}{ Near field Communication } & \\
\hline & & \multicolumn{3}{|c|}{ Social Networks } & \\
\hline
\end{tabular}

Fig 2: Technologies associated with IoT.
As a result, these IoT related technologies have also made a large impact on new Information and Communication Technology(ICT) and Enterprise System Technologies (see fig.3) [18].

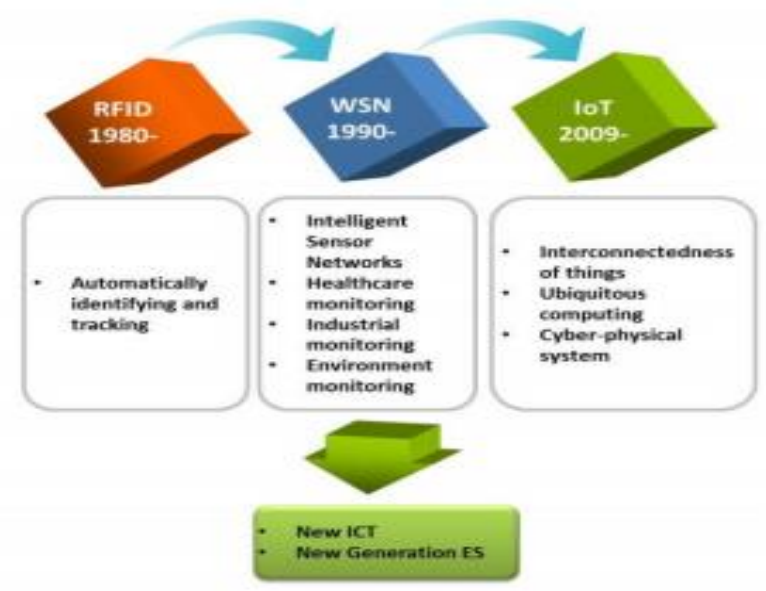

Fig 3: IoT technologies impact on ICT and enterprise systems.

\section{Enabling Technologies:}

In the real-world technology, the concept of IoT is probably the combination of several enabling technologies which are listed below.

\subsection{Identifying and Tracking Technologies:}

Initially, RFID system is used in identification and tracking of objects, people, things etc. RFID systems are increasing in usage because of its tracking and tracking of devices, objects used many industries, health care, monitoring, logistic, supply chain management etc [6] [19], as well as RFID systems are also used by manufacturers, distributors, retailers in many industries [7][8]. A physically system consists of Reader, tag, and Antenna. Physical parts of system are shown below fig4.

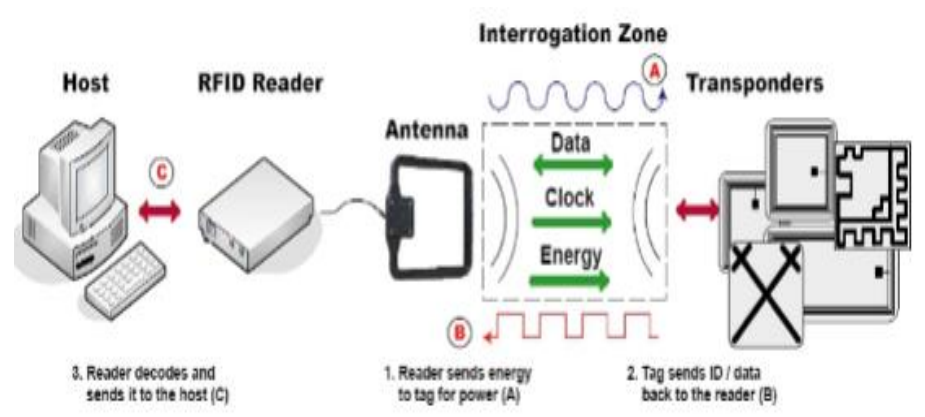

Fig 4: RFID System

RFID Reader: It generates radio waves ranging from 1 to 100 inches on the basis of radio frequencies. RFID tag detects activation signals of a reader. RFID Reader will communicate with all tags within the range [20]. RFID Reader process multiple items at once. Transmission of signals may occur in a range of several frequencies bands.

RFID Tag: It consists of a chip and an antenna. The chip consists of unique number and information of tag's memory type that can be read-only, read-write, write once and read many. Antenna transmits information from chip to reader. The read range depends on antenna largeness. RFID Tags are of the different type listed in fig 3 .

Copyright $\odot 2018$ Authors. This is an open access article distributed under the Creative Commons Attribution License, which permits unrestricted use, distribution, and reproduction in any medium, provided the original work is properly cited. 


\begin{tabular}{|l|l|l|l|}
\hline & $\begin{array}{l}\text { Passive } \\
\text { Tags }\end{array}$ & $\begin{array}{l}\text { Semi- } \\
\text { Passive } \\
\text { Tags }\end{array}$ & $\begin{array}{l}\text { Active } \\
\text { Tags }\end{array}$ \\
\hline $\begin{array}{l}\text { On board } \\
\text { powrer supply }\end{array}$ & $\begin{array}{l}\text { No (From } \\
\text { Reader) }\end{array}$ & $\begin{array}{l}\text { Yes } \\
\text { (Internal } \\
\text { Battery) }\end{array}$ & $\begin{array}{l}\text { Yes } \\
\text { (Tnternal } \\
\text { Battery) }\end{array}$ \\
\hline $\begin{array}{l}\text { Transmission } \\
\text { range }\end{array}$ & $\begin{array}{l}\text { Short (up } \\
\text { to } 6.096 \\
\text { meters) }\end{array}$ & $\begin{array}{l}\text { Medium } \\
\text { (up to } 30.48 \\
\text { meters) }\end{array}$ & $\begin{array}{l}\text { Long (up } \\
\text { to } 228.6 \text { m) }\end{array}$ \\
\hline $\begin{array}{l}\text { Communication } \\
\text { pattern }\end{array}$ & $\begin{array}{l}\text { Pasive } \\
\text { Cost }\end{array}$ & Pasive & Proactive \\
\hline Type of \\
memory
\end{tabular}

Fig 5: Different types of RFID Tags.

RFID passive tag does not contain their own power source they simply rely on the reader. Passive tags harvest the energy from nearby RFID Reader. The cost is based on the memory used, the antenna design and other requirements. Where RFID active tag contains their own power supply such as batteries that communicate with the reader. The longer distance covered by tag is based on battery power. RFID active tag contains more hardware equipment than passive tags. The cost of passive tags is expensive than active tags. Semi-passive tags do not initiate communication with the reader but contains the batteries to perform a different function.

As RFID device results are more accurate in tracking, tracing, identification many research using this RFID devices in many safety and security applications. RFID is used in women security for identifying the location along with using GSM technology [21].

\subsection{Communication Technologies and Networks in- volved in IoT:}

Communication Technologies play a crucial role in Wireless Networks. In IoT context there is number of heterogeneous networks, such as WSN, WMN, WLAN etc., help IoT to exchange information. IoT Networks make use of internet support for information exchange and data communication. There is few cross layers protocol for the wireless network such as WSAN and Adhoc network. Communication technology in IoT has low bandwidth, and power consumption. some of the major technologies utilized by IoT devices for communication are:

3.2.1 ZigBee: ZigBee is IEEE 802.15.4 industry standard maintained by the ZigBee Alliance. It is low power, low-cost, and low throughput technology. It is used in low data rate application that requires low battery life and secure networking. It consists of some unique functionalities with long battery life [22]23]. It transmits data over a long distance of up to 10 to 20 meters approximately. ZigBee is used in embedded sensing, medical data collection, home automation, industrial control, smart energy devices, HVAC, and security etc. An application level gateway is required by ZigBee network to connect to IoT. In ZigBee network the gateway participates as one of the nodes and parallelly runs a TCP/IP stack and application over ethernet/wi-fi.

3.2.2 Bluetooth: A wireless protocol designed for low power consumption with cost-effective. It is standardized by IEEE 802.15.1, but no longer standard now it is managed by SIG (Blue- tooth Special Interest Group). In case of IoT devices would like to interconnect with IoT (sensor \&actuator) with limited power resources. Bluetooth does not have direct connectivity to the internet. It connects the wireless devices up to $10 \mathrm{~m}$ to a smart phone which act as an internet gateway. If once a connection is established it maintains the link even there is no data flow.

3.2.3 Z-WAVE: It is wireless communication protocol of low power consuming and primarily used in home automation. Z-wave is developed by ZenSys in 2001 and promoted by Z-wave alliance. $\mathrm{Z}$-wave operates at $800-900 \mathrm{MHz}$ radio frequency range. Z-wave consists of one hub controller and a second type which reply to the controller. Z-wave is mainly used in residential appliances such as lighting control, thermostats, window, locks, swimming pool and garage door openers.

3.2.4 6LoWPAN: 6LoWPAN is IEEE 802.15.4 standard with low power wireless personal area network that supports ipv6 network. [24]. It is low power consumption device with limited processing capabilities is able to participate in the IoT. It uses mesh network where each node has its own address of ipv6 and connect directly to the internet.

3.2.5 WI-FI: It is wireless fidelity, based on the IEEE 802.11 standard to connect devices to the internet. It handles high quantities of data and provides fast data transfer. Wi-fi network is available within the range of $50 \mathrm{~m}$ approximately and connects to phone, laptops, devices. To transmit and receive data at high speed it uses radio technologies. Wi-Fi uses high power consumption compared to some other standards. Wi-Fi is a simple, easy and less cost to connect to the internet without using any wires. Its popularity increases because of its cost effective and freedom it gives to users. $\mathrm{Wi}-\mathrm{Fi}$ becomes a ubiquitous technology for wireless internet connectivity [23]. High power and complexity barriers are reduced by new silicon devices and modules and enable Wi-Fi integration into emerging IoT applications and devices based on batteries.

\begin{tabular}{|c|c|c|c|c|c|}
\hline Standard & Bluetooth & ZigBee & Wi-Fi & 6LOWPAN & Z-Wave \\
\hline IEEE Spec. & $\begin{array}{c}\text { IEEE } \\
802.15 .1\end{array}$ & $\begin{array}{l}\text { IEEE } \\
802.15 .4\end{array}$ & $\begin{array}{c}\text { IEEE } \\
802.11 \mathrm{a} / \mathrm{b} / \mathrm{g} / \mathrm{n}\end{array}$ & $\begin{array}{c}\text { IEEE } \\
802.15 .4 \\
2006\end{array}$ & z-Wave alliance \\
\hline Topology & Star & Mesh, Star, Tree & Star & Mesh, Star & Mesh \\
\hline Bandwidth & 1 Mbps & $250 \mathrm{Kpbs}$ & Upto 54 Mbps & $250 \mathrm{Kbps}$ & $900 \mathrm{MHz}$ \\
\hline $\begin{array}{c}\text { Power } \\
\text { Consumption }\end{array}$ & Very Less & Very Less & Low & Very Less & Very low \\
\hline $\begin{array}{c}\text { Max data rate } \\
\text { (M bit } / \mathrm{s})\end{array}$ & 0.72 & 0.25 & 54 & $800 \mathrm{~m}$ (SubGHz) & 9600 bits or 40 Kbits \\
\hline Bit time $(\mu s)$ & 1.39 & 4 & 0.0185 & - & - \\
\hline Range & $<30 m$ & $10-300 \mathrm{~m}$ & $4-20 \mathrm{~m}$ & $800 \mathrm{~m}$ (SubGHz) & $30 \mathrm{~m}$ \\
\hline Spectrum & $2.4 \mathrm{GHz}$ & $2.4 \mathrm{GHz}$ & $2.4-5 \mathrm{GHz}$ & $2.4 \mathrm{GHz}$ & $2.4 \mathrm{Ghz}$ \\
\hline $\begin{array}{l}\text { Channel } \\
\text { Bandwidth }\end{array}$ & $1 \mathrm{MHz}$ & $\begin{array}{c}0.3 / 0.6 \\
\mathrm{MHz}, 2 \mathrm{MHz}\end{array}$ & $22 \mathrm{MHz}$ & $\begin{array}{c}868-868.6 \\
\mathrm{MHz} \text { (EU) } \\
902-928 \mathrm{MHz} \\
\text { (NA) } \\
2400- \\
2483.5 \mathrm{MHz} \\
\text { (WW) }\end{array}$ & $868 \mathrm{MHz}$ \\
\hline
\end{tabular}

Fig 6: Comparison of Communication Technologies

\section{IoT Architecture:}

4.1 Device Layer: It is physical device layer where physical devices like sensor/actuators, RFID will connect to the internet. It is the bottom layer of IoT architecture will transmit the data to next upper layer [25] [26]. Various types of devices are considered as IoT devices which attaches to the internet for communication some of the direct connection devices with internet are Arduino, Arduino Yun, ZigBee devices via ZigBee gateway, Bluetooth devices via mobile phone, Raspberry Pi connected via Ethernet or Wi-Fi. Various sensors are used for identify other smart objects in 
the environment. Each device needs a universally unique identifier(UUID) which is easily identified.

\subsection{Communication Layer (Network\& Transport layer):}

Networking layer connects all the things for sharing information with other things. This layer transfers data from sensing/devices layer to upper layer. It securely transfers the confidential data from sensor/actuator to information processing system by using Wi-Fi,3G,4G, Satellite and various Wireless Sensor Networks, WLAN, etc., Network layer is capable of gathering all the information business system, transportation system, healthcare system, ICT etc., Things will provide different services which are deployed on heterogenous network and are brought for communication into service network. It also involves Quality of Service management and controls according to the user/application requirements. In IoT architecture, the network layer needs to address issues such as QoS requirements, security, privacy, network management technologies for heterogeneous networks, service discovery, data signal processing. This layer has transporting capabilities to upper layers. The transport session uses IPv4, IPv6 protocol and some HTTP, FTP, MQTT [28] [27] Dos protocol for communication. Different forms of a network like the public, private, hybrid is built to support communication requirements for security, bandwidth, and latency.

4.3 Service Layer: It relies on middleware technology. Service layer performs following functionality including the exchange of information and storage, data management, search engine, and communication. It provides functionalities for services offered by the network object to build applications. In this layer, only services are visible. A service repository is maintained and specific services will be executed at run-time to build composed services. This layer includes some proposals of middleware such as functionality related to QoS \& lock management and some semantic functions. This layer will enable services like object discovery, services configuration, and status monitoring. It will also enable the deployment of new services at runtime for satisfying the user/application needs.

4.4 Application Layer: It acts as an interface between system functionalities and users. It uses standard web service protocols and service composition technologies and languages and provides a perfect integration between application and distributed system. As there are numerous devices connect to the network, the application can be from different areas like manufacturing, logistics, industries, public safety, healthcare, food, environment, retail etc., the responsibility of this layer is event detection and perform user required function.

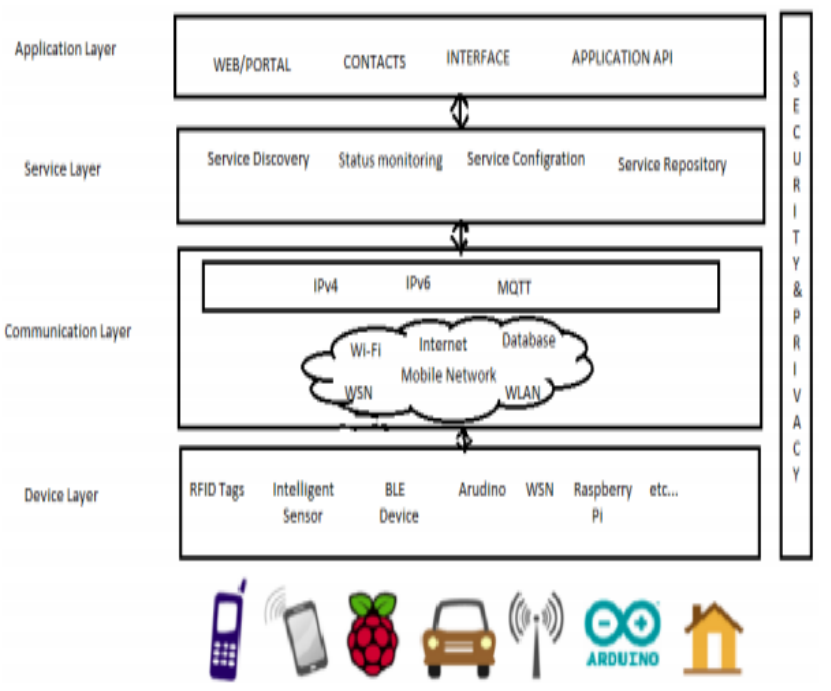

Fig 7: IoT Architecture

\section{Role of IoT in Industries (IIoT):}

IIoT is Next generation in Industries. Industrial Internet of Things is a part of a large concept of IoT. Use of IoT to the manufacturing industry is called the IIoT (or industrial Internet or Industry 4.0). As we known, IoT include consumer level devices like heart monitoring fitness bands, smart appliances, etc., whereas IIoT is to think of Industry internet connecting machines and devices in industries like oil \& gas, power generation, and health care, where system failure and unplanned downtime can result in hazardous condition. Below fig shows the CIoT \& IIoT. IIoT will enable industries to do predictive analytics in order to detect corrosion inside a refinery pipe and provide real-time production data in a plant. It is mentioned that integration of Information technology and operational technology is crucial in Industrial Internet of Things [29]. There are different industry bodies have different approaches as you will discover, but the two main ones are platform industry 4.0 (restricted only to manufacturing) and the industrial internet consortium. Industry 4.0 says we are entering the fourth industrial evolution, the industrial internet speaks about the third industrial innovation wave. Industry 4.0 describes a new industrial revolution with a focus on automation, innovation data, cyber physical system, process \& people.

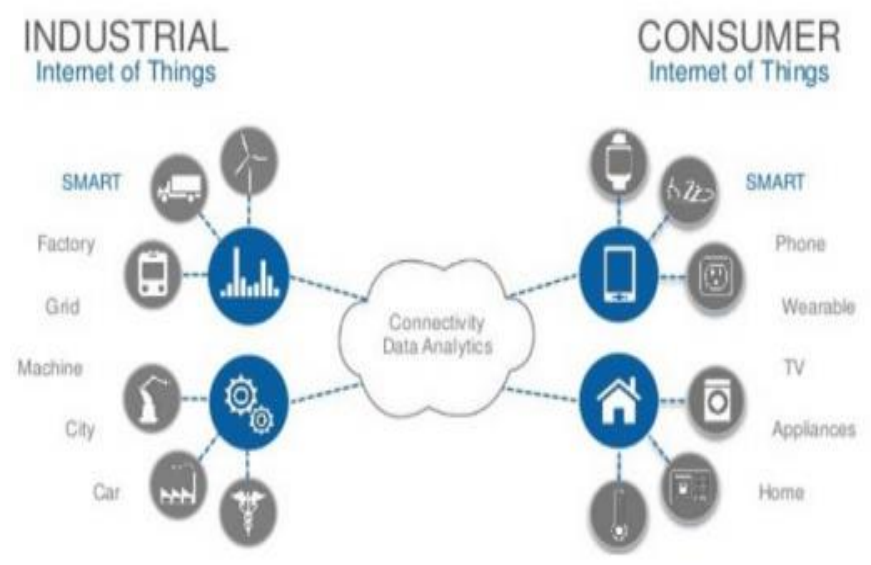

Fig 8: Applications of CIoT \& IIoT.

\subsection{Benefits, Protocols \& Challenges of IIoT:}

IIoT improves efficiency scalability, cost \& time saving for indus-trial organizations. A lot of organization are benefited by consider-ing the IIoT application. It allows organisation to get an overview of their enterprise functions, which will help them to take well decision. Among all other communication protocol, the MQTT [30] [31] is quickly making its appearance as the standard for IIoT due to its light weight overheads, bidirectional capabilities etc; security \& interoperability are two main challenges surrounding the IIoT implementation.

\section{Application of IoT:}

\subsection{Personal \&Home:}

Smart home/office are the popular IoT application [32] [33]. The Products are affordable and readily available to consumers. Many products, hundreds of products are available in the market through which the consumer is more connected to their lives even before such products example (Amazon Echo) which allows the user to control the data/things with their voice. Many electronic appliances like a washing machine, refrigerator, micro waves, air conditioner etc., and simple devices like light bulbs can thus be monitored and controlled. A smart phone app can access, control and monitor the device data thus resulting in better home and energy management. 


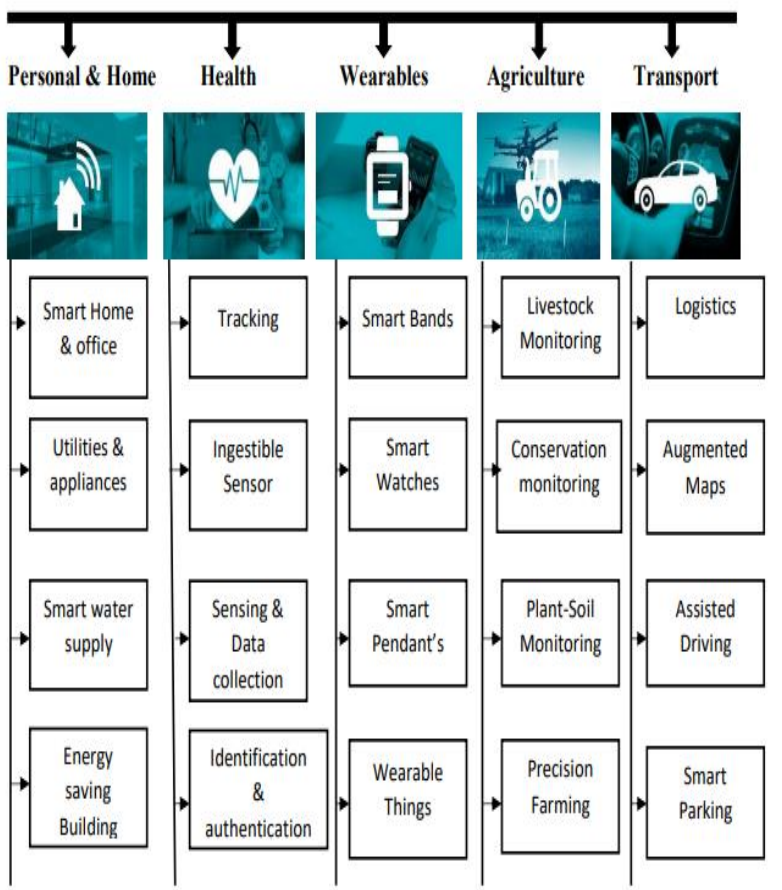

6.2 Health:

IoT makes medical equipment more efficient through sensors which acquire patient data and reduce human errors. It provides an accurate collection of data, reduces system cost, provides real-time information which aids them to make upto-date decisions and offer the necessary treatment. Tracking is most frequently used for continues inventory location tracking and tracking materials and help to prevent left-ins during surgery. The ingestible sensor is smart capsules which upon swallowing, gets activated by electrolytes with in the body. It transmits the signal to device on user's body and send the data to the phone. Identification of patient, helps to reduce incidents like wrong drug/dose/time/procedure Authentication \& Identification is mainly used for security concern, in order to provide access to staff for patient issues. [32] [34]

\subsection{Wearables:}

Wearables are the hall mark of the Internet of Things and the most ubiquitous of its implementation to date. Wearables like various smart wrist wear, hearables and smart glass, smart bands etc., are getting closer and bring exceptional values to our lives [32]. These wearable devices enable instant interoperability, profile management, notification, data collection, security alerts and other features to our smart phones.

\subsection{Agriculture:}

Precision Agriculture is a concept where farmer accesses the parameters of his farm and control the parameters either manually or automatically. Parameters like reading and writing are digitally done. Area of the plot for crop plantation is monitored and controlled [35]. Livestock monitoring is all about animal's husbandry. IoT wireless sensor helps the ranchers to gather data regarding the health, location, and wellbeing of their cattle. The data will recognize the sick animals in the cattle and can be moved from the herd and reduce the number of sick animals from the cattle. It also helps the ranchers by where the cattle are located and lower the labour costs. Plant \& soil monitoring will lead to the fantastic return on farmers investments. The greater uses of agriculture IoT are sensing for soil moisture \& nutrients, maintaining water usage for optimal plant growth and determining fertilizer profiles based on soil chemistry and many more [36].

\subsection{Transport:}

IoT is already a boon to transport and logistic sector. IoT will provide a real-time visualization of entire transport fleet and operations. Transport and logistic sector will be the leading adopters of this revolutionary technology to ensure security, efficiency and keeping pace with challenges.

\subsection{Logistics:}

IoT technology can be applied in the logistic industry as well as freight goods. Transport continues to be permeated with challenges such as weather, distance, thefts etc on a day to day basics. With machine-to-machine(M2M) communication and global positioning system(GPS) capabilities the real-time monitoring of vehicle in motion, position and rest of its contents can be tracked. It is not about just tracking and arriving vehicle at right time business also add sensor inside the vehicle to ensure the package is at the right temperature. IoT can ensure the biggest and most immediate support of efficient monitoring to avoid predictable delays with this vehicle carrying perishable or temperature sensitive goods can be tracked, maintained at a right temperature, secure and not damaged [37]. It can also add a sensor to third-party devices like fuel pumps, to ensure that the customer is getting all they require from the supplier. Once an order comes in the IoT system is capable of obtaining the stock, packaging labelling and sending it much faster or quicker than a human workforce.

\subsection{Assisted Driving [38]:}

Vehicles are implemented with processing power, sensor, actuators and will provide data to the driver or passenger of the vehicle to provide better navigation. Will see in near future, the development of an autopilot which detect pedestrians automatically and take oblique steering to avoid the collision. To avoid collision and accidents, safety features are designed to alert the driver to avoid potential problems by implementing safe guards and taking over control of the vehicle. IoT will provide the connection between and among vehicle. communication among vehicle enables to communicate with their technicians (driver) anytime, anywhere, allowing them to be proactive with in-field repairs, maintenances etc. onboard sensor can help to maintain vehicle health by monitoring their status and alert the driver if any abnormality is detected. The up to minute traffic information can be gathered and this data can help drivers to slow down if an accident may have taken place at a spot head.

\section{Operating systems used in IoT devices:}

The operating system for IoT devices is classified into two categories, the high-end IoT device, and low-end IoT device. The highend IoT device will have better capabilities and performance to run software based on the traditional operating systems such as Linux or BSD. To run this traditional operating system low-end devices are less resource constraint. While the low-end IoT devices include Arduino [39], zolertine [41], IoT lab M3 nodes [42], Econotag [40], open-mote nodes [43] and Telos B motes [44] whereas single board computer such as Raspberry $\mathrm{Pi}$ and smartphones are examples of high end IoT devices. The IoT OS is an evolution of embedded OS because it brings the additional set of constraints that to be addressed. Today in the market a mixture of closed source and open sources OS exists. A clear view on these OS will explain latter in this paper

\subsection{Parameter Requirements for IoT OS:}

\subsubsection{Memory Footprints:}


IoT devices are resource constraint in requirements of memory with only a few kilobytes of memory while PC's laptops, smartphones, tablets provide Giga and Tera bytes of memory.

\subsubsection{Network connectivity \& protocol support:}

The IoT devices can interconnect and communicate with one another or with the internet. These connections are achievable by providing various connectivity protocol support like wi-fi, cellular, Bluetooth, etc. the connectivity process should be simplified by operating systems. Contrary to WSN [47] [48] scenario it is expected that IoT devices seamlessly integrated and communicate with end to end machine via, the internet [46]. The evolution of Linux over the years indicates the desirable that the OS can cater for multiple network stacks and continues for network stack evolution.

\subsubsection{Energy Efficiency:}

Most of the IoT devices will run on batteries or other constraint energy resources. Many devices are required to work for years with single battery [49] charge example are smart meters, home/building automotive devices. As the number of IoT devices are expected to be deployed the energy efficiency is more required To provide better energy efficiency, the IoT OS should have features like putting the device in sleep mode as often as possible and save energy for upper layers and allowing the minimum number of periodic tasks to be executed. The IoT OS should be highly energy efficient.

\subsubsection{Security:}

The devices are connected to the Internet, with high security and privacy standards. Security challenges in IoT like data integrity, authentication, and access control in various layers of IoT architecture. Thus, the challenges for IoT OS are to provide such mechanisms like security, protocols, cryptographic libraries etc., to meet the strict security requirements. The IoT OS should also provide a mechanism for software updates on already deployed IoT devices and to use open source os as much as required [50].

\subsection{Open Source Operating System:}

\subsubsection{CONTIKI OS [51] [52]:}

It is an open source operating system runs on low power wireless devices. Contiki OS is designed with a small amount of memory for microcontroller and freely used by both in commercial and non-commercial systems. It is written in c language, protothread and event driven are programming models used. It supports monolithic architecture and uses the co-operative scheduler. Contiki is highly portable, multi-tasking OS for memory efficient network embedded system and wireless sensor networks. It is BSD license and available on GitHub. It is oldest among all open sources OS's for constrained nodes developed since 2002. It has been used in road tunnel fire monitoring, intrusion detection, wild life monitoring and in surveillance networks.

\subsubsection{Tiny OS [53]:}

It is most used an open source OS's so far used for constrained nodes, with Contiki. It is developed since 2000 and oldest among all open sources along Contiki. It follows an event-driven programming model supports co-operative scheduler with monolithic architecture. In tiny OS a set of required components are combined together to build a single, static binary to expose one or more interfaces and communicate with commands and events. Tiny OS is written in c-dialect called nesC. It is the BSD license OS and its source code is available on GitHub. It is embedded, the component-based OS for low end devices used in Wireless SN and supports hardware platform of Arduino, Iris mate, Sunspot, Xbee etc; and support BLIP network stack.it uses in the applications like smart building, PAN (personal area networks), sensor networks ubiquitous computing, smart meters.

\subsubsection{RIOT OS [54] [55]:}

It is micro kernel-based OS with various software requirements for IoT devices. RIOT features the implementation of several network stack like 6LoWPAN stack, a port of information centric network stack CCN-Lite [57], a port of 6TiSCH stack open WSN [56]. Similarly, as what is experienced on Linux. RIOT provides developer friendly API across all platforms. It uses less than $5 \mathrm{~Kb}$ of rom and less than $2 \mathrm{~kb}$ of RAM. It is written in c language, applications can also implement in $\mathrm{C}++$, the source code is available under LGPLv2. RIOT supports multi-threading programming model and uses the pre-emptive scheduler.

\subsubsection{Free RTOS [58]:}

It is popular RTOS for embedded devices which have been ported to many micro controllers.it uses the pre-emptive scheduler for multi-threading programming model.it supports micro kernel RTOS architecture its source code is available under free RTOS license with a modified GPL that allows user proprietary code to remain closed source whereas kernel itself as open source. For internet connection third-party network stack is used it don't have its own network stack. Its design is simple with small memory footprints low overheads and fast execution. Free RTOS is developed professionally with good quality controlled, robust and supported to use even in commercial application.

\subsubsection{UCLINUX [59]:}

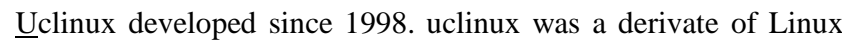
2.0 kernel intended for the micro controller without MMU and with small memory footprints then Linux. uclinux benefits from features set of Linux like a full TCP/IP stack, excellent file system support and including API's. Its source code is available on Source forge.

\subsubsection{NuttX [60]:}

NuttX is primarily known as the most common RTOS for open source drones running on APM, PX4, UAV platforms. It has full POSIX and ANSI complains and support x86\&cortex A5\&A8 platforms.it has micro kernel architectures and support monolithic as well. It uses pre-emptive scheduler and has multi-threading programming model.it is written in c code and source code is available under BSD license. Its network stacks include support of IPV4\&IPV6with various upper layer protocols.

\subsection{CLOSED SOURCE OS:}

\subsubsection{ThreadX [61]:}

ThreadX is express logic's advanced industrial grade real-time os designed for embedded, and IoT applications. It is the ideal choice for most of the embedded applications. ThreadX is based on micro kernel RTOS, supports multi-threading and uses pre-emptive schedules. ThreadX has small footprints of $2 \mathrm{~kb}$ of instruction area and $1 \mathrm{~kb}$ of RAM. It provides simple, easy to use and fast execution environment. It supports popular embeded development tools, IAR's embedded workbench etc., It supports features like preemption threshold, scheduling, event chaining, execution profiling, performance metrics including its pico- kernel architecture. Its network stack, GUI, file system, USB support are separately purchased for add-on additional features.

7.3.2 Vx Works [62]: Vx works is proprietary \& customizable real-time operating system developed by Wind River and now 
owned by Intel. It is initially developed since 1987 and it is the monolithic kernel that supports mostly ARM platform \& intel platform. Vx works support IPV6and lacks the support for 6lowpan stack.

7.3.3 Pike OS [63]: It is hard real-time operating system developed by SYSGOAG. It offers a separation kernel-based hypervisor with multiple partitions of another operating system (Guest OS ). Pike OS is the clone of L4 microkernel family, it is originally called P4. It enables the user to execute safety, security, and highquality application on the same platform. Features like reduce time to market via standard development and verification tools and support the wide range of guest OS type[API's].

7.3.4 Nucleus RTOS [64]: Nucleus is real-time OS offered by embedded software division of mentor graphics which supports 32 $\&$ 64bit embedded platform. The latest version supports 64bit ARM architecture and includes features like process model, safety certification, power management and complex heterogenous multicore SOCs. Its source code is written in clanguage it has limited memory foot prints of $<10 \mathrm{kbs}$ for both data and code. It supports both high-end MPU's, MCU's, DSP's, supports for SMP and AMP. It enables $\mathrm{C}++$, POSIX and micro ITRON interfaces.it supports POSIX network stack and provide easy use of application interface. The recent release of nucleus support COAP, MQTT, 6LoWPAN, and HTTPS.

7.3.5 Window CE [65]: Window CE is sub family of window OS developed by Microsoft since 1996. It is the real-time operating system with small memory footprints. The devices with window CE can have less than a megabyte of memory, it supports x86, 32bit ARM processor with BSP (Board Support Package) directly. It is written in c language and it is hybrid kernel type.

7.3.6 Liteos Huawei: Huawei release a Liteos, an operating system for IoT devices. It is light weight with $10 \mathrm{~kb}$ of memory which can run on AA batteries up to five years with very little power consumption suitable for wide range of hardware including ARM cortex embedded processor, microcontrollers.it provides an open IoT ecosystem for partners to quickly develop IoT products $\&$ accelerate IoT development. It simplifies the IoT devices connectivity, makes services smarter, provides better data protection, delivers excellent user experience. It allows fast start up connectivity and very secure. As IoT is evolving drastically and having ubiquitous nature we can make use in numerous fields. in this paper, we are providing one such way how IoT is helpful to women in society. Currently, there are many existing devices and app which will provide safety to women just in one click.

\section{EXISTING SYSTEMS:}

VithU app: An emergency and user-friendly app can be downloaded on Android phones which are initiated by a popular Indian Crime Television series "Gumrah" aired on Channel [V]. Clinking twice the power button of your smart phone, it consequently beings sending messages to your contacts for every 2 minutes with a link of providing latitude and longitude location.

SHE: It is underarm garments implemented with an electronic device actuated with the sensor and electric shock circuit board for women which generates a $3800 \mathrm{kv}$ to help the victim to protect and escape from atrocities [66]. It can also send text messages to parents and local police station.

AESHS (Advanced Electronics system for Human Safety): A safety device which can be tracked and monitor the victim's location using the GPS facility. Stun Gun: It helps the victim to escape from the attacker by giving him an electric shock. It pumps about 700,000 volts into attacker's body which gives the victim a sample chance to escape the scene, the shock weakens the attacker temporarily. This device is easy to carry in handbags.
Stun Gun: It helps the victim to escape from the attacker by giving him an electric shock. It pumps about 700,000 volts into attacker's body which gives the victim a sample chance to escape the scene, the shock weakens the attacker temporarily. This device is easy to carry in handbags.

Jivi 2010: A feature in Jivi Mobile with SOS button mainly aimed at women in stress. In case of emergency, user need to long press the button and the phone starts calling 5 pre-set contact numbers one after other if any number is busy and the call was not taken, an SMS will be sent to the number. After this the phone dials another number automatically from the pre-defined list for immediate help.

Fight Back: This is an app similar to ones listed above. Its unique functionality is Facebook status update and also providing SMS and email option in distress situations.

\section{PROPOSED SYSTEM:}

The system aims at designing a security system for women. This project presents an intelligent tracking system for women and the message regarding the location of the system is sent to the predefined contacts. Keeping in a mind that if a victim is not in a position to take an initial step to press a button on safety device, we are providing a microphone output is fed to the Raspberry Pi processor. Whenever women ask for help through the microphone the GPS receiver tracks the location of the women and messages are sent to predefined contacts through GSM and we are also adding the features like web camera for live streaming and takes snaps that are sent to predefined mail-ids.

\subsection{Methodology:}

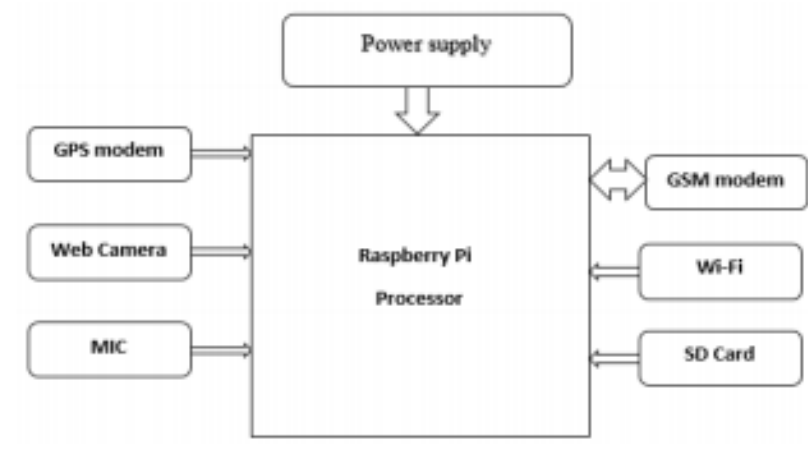

Fig 9: Women Safety System

\subsection{Major Building block of this Project are:}

9.2.1 Power Supply: A power switch that goes up to 2.5 Amps allowing your pi to power even more powerful devices over USB ports.

9.2.2 Raspberry Pi 3 Processor: Raspberry Pi 3 is a series of Identity-Card sized single board computer with having similar capability of what your PC does like spread sheets, word processing and playing high definition video games. It can run on both Linux \& windows 10voperating system. Raspberry pi 3 is based on Broadcom BCM 2837, an ARM CortexA53 1.2GHz Quadcore Processor System on Chip (SOC) with 802.11bgn Wireless LAN and Bluetooth

9.2.3 GSM Modem: Itis a specialized type of modem which accepts a SIM Card and operate just like a mobile phone. The SIM 900 is a complete Dual Band GSM/GPRS module in a SMT type, the SIM 900 delivers GSM/GPRS 900/1800 MHz performance for voice, SMS, data with low power consumption. It communicates with controllers via AT Commands, it has small configuration of 
$24 \mathrm{~mm} * 24 \mathrm{~mm} * 3 \mathrm{~mm}$ and can fit in almost all the space requirements in your application.

9.2.4 GPS Modem: Global Position System(GPS) is able to determine the longitude and latitude of current location of user. The current date, time, altitude, speed and travel direction among the other related data are provide by the module. The module can support 57 channels. It can be used in applications like navigation, tracking system, mapping and robotics, fleet management.

9.2.5 Wi-Fi: The wi-fi module contains SOC with integrated TCP/IP protocol stack that allows your microcontroller to access the wi-fi network.

9.2.6 Microphone: It recognizes the voice of the user and simultaneously perform audio recording and send messages to pre-set contacts.

9.2.7 Web Camera: This module is used for live streaming and capture the images and the recorded video is further used as proof. 9.2.8 Hard disk (SD Card): All the recorded data and captured images are stored in SD card which can be used as back up for investigation purpose.

9.2.8 Hard disk (SD Card): All the recorded data and captured images are stored in SD card which can be used as back up for investigation purpose.

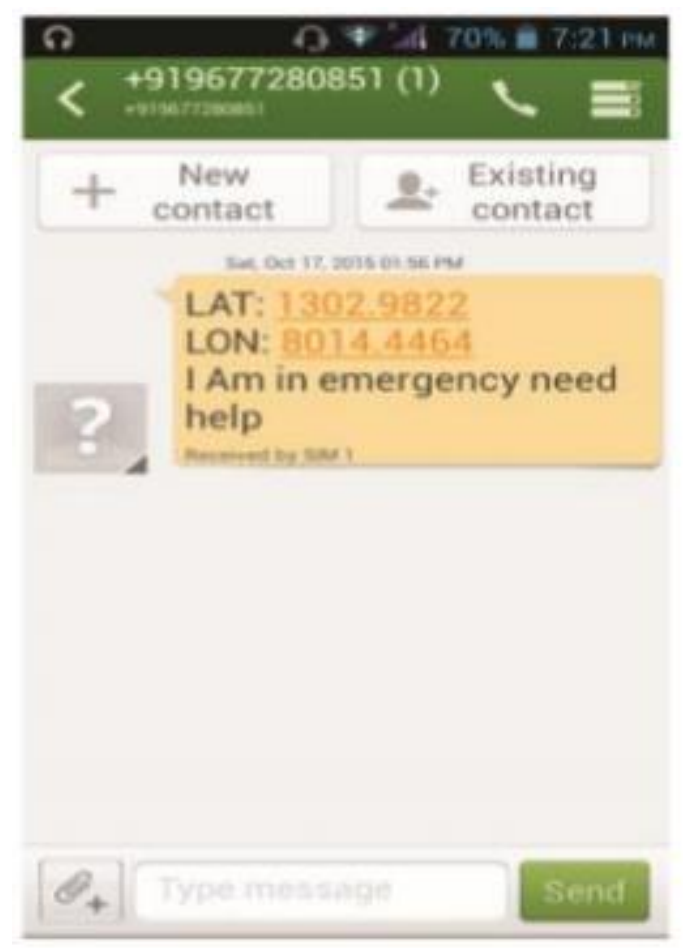

Fig 9: Showing the Latitude and Longitude Location of sender

\section{CONCLUSION:}

This paper gives the reader an overall view of IoT (Internet of Things) how it drastically changes the people's day to day life and one such real-time application device for providing women security using IoT technology. The proposed design will deal with Critical issues faced by women in recent past and helps to solve them technologically using Compact equipment and ideas. The system can overcome their fear that scars about their safety and security. This system can further be researched with new innovative ideas and can be implemented in different areas of security and surveillance.

\section{References}

[1] K. Ashton, "Internet of Things," RFID Journal, June 22, 2009

[2] R. van Kranenburg, E. Anzelmo, A. Bassi, D. Caprio, S. Dodson, and M. Ratto, "The Internet of things," in Proceedings of 1st Berlin Symposium on Internet and Society, pp. 25-27, 2011.

[3] X. Jia, O. Feng, T. Fan, and Q. Lei, "RFID technology and its applications in the Internet of Things (IoT)," in Proceedings of the 2nd IEEE International Conference on Consumer Electronics, Communications and Networks (CECNet), April 21- 23, 2012, pp.1282-1285.

[4] C. Sun, "Application of RFID technology for logistics on the Internet of Things," AASRI Procedia, vol.1, pp.106-111, 2012.

[5] E. W. T. Ngai, K. K. Moon, F. J. Riggins, and C. Y. Yi, "RFID research: an academic literature review (1995-2005) and future research directions," International Journal of Production Economics, vol.112, no.2, pp.510-520, 2008.

[6] Ashton, K.: That 'Internet of Things' Thing. RFID Journal 22, 97 114 (2009)

[7] Auto-Id Labs

[8] The EPC global Architecture Framework, EPC global Final Version 1.3, Approved 19 March 2009, www.epcgloballinc.org.

[9] Luigi Atzori, Antonio Iera, Giacomo Morabito "The Internet of Things: A Survey", Article in press on Computer Networks, May 2010

[10] S. Li, L. Xu, and X. Wang, "Compressed sensing signal and data acquisition in wireless sensor networks and Internet of Things," IEEE Transactions on Industrial Informatics, vol.9, no.4, pp. 2177-2186, 2013

[11] W. He, and L. Xu, "Integration of distributed enterprise applications: a survey," IEEE Transactions on Industrial Informatics, vol.10, no. 1, pp.35-42, 2014

[12] D. Uckelmann, M. Harrison, and F. Michahelles, "An architectural approach towards the future internet of things", in Architecting the Internet of Things, pp. 1-24, Springer, 2011

[13] S. Li, L. Xu, X. Wang, J. Wang, "Integration of hybrid wireless networks in cloud services-oriented enterprise information systems," Enterprise Information Systems, vol.6, no.2, pp.165-187, 2012 .

[14] Li. Wang, L. Xu, Z. Bi, Y. Xu, "Data Filtering for RFID and WSN Integration," IEEE Transactions on Industrial Informatics, vol.10, no.1, pp 408-418, 2014.

[15] L. Ren, L. Zhang, F. Tao, X. Zhang, Y. Luo, Y. Zhang, "A methodology towards virtualization-based high-performance simulation platform supporting the multidisciplinary design of complex products," Enterprise Information Systems, vol.6, no.3, pp.267290, 2012

[16] F. Tao, Y. Laili, L. Xu, L. Zhang, "FC-PACO-RM: a parallel method for service composition optimalselection in cloud manufacturing system," IEEE Transactions on Industrial Informatics, vol.9, no.4, pp.2023-2033, 2013.

[17] Q. Li, Z. Wang, W. Li, J. Li, C. Wang, R. Du, “Applications integration in a hybrid cloud computing environment: modelling and platform," Enterprise Information Systems, vol.7, no.3, pp.237- 271, 2013

[18] Li Da Xu, Wu He, and Shancang Li "Internet of Things in Industries: A Survey", IEEE Transaction on Industrial Informatics, Vol.10, No.4, November 2014

[19] M. K. Lim, W. Bahr, and S. Leung, "RFID in the warehouse: a literature analysis (1995-2010) of its applications, benefits, challenges and future trends," International Journal of Production Economics, vol.145, no.1, pp.409-430, 2013.

[20] www.wikipedia.com

[21] Shaik Mazhar Hussain1, Shaik Jhani Bhasha2: Design of women safety system using RFID, 8051microcontroller and GSM-based technology a prototype June (2014)

[22] ZigBee - The Internet of Things, http://www.vesternet.com/zigbee (accessed on November 2012)

[23] Lee, J.S., Su, Y.W., Shen, C.C.: A comparative study of wireless protocols: Bluetooth, UWB, ZigBee, and Wi-Fi. In: 33rd Annual Conference of the IEEE Industrial Electronics Society (IECON 2007), pp. 46- 51 (November 2007)

[24] Ee, G. K., Ng, C. K., Noordin, N. K., Ali, B. M.: A Review of 6LoWPAN Routing Protocols. In: Proceeding of Asia Pacific Advanced Network (2010)

[25] Z. Yang et al., "Study and application of the architecture and key technologies for IoT," in Proc. ICMT, 2011, pp. 747-751. 
[26] M. Wu, T. J. Lu, F. Y. Ling, J. Sun, and H. Y. Du, "Research on the architecture of Internet of Things," in Proc. 3rd ICACTE, 2010, pp. V5-484-V5-487.

[27] D. Locke, "MQ telemetry transport (MQTT) v3. 1 protocol specification," IBM developer Works, Markham, ON, Canada, Tech Lib., 2010. [Online]. Available: Http://Www.Ibm.Com/Developerworks/ Webservices/Library/Ws-Mqtt/Index.Html

[28] U. Hunkeler, H. L. Truong, and A. Stanford-Clark, "MQTT-SA publish/subscribe protocol for wireless sensor networks," in Proc. 3rd Int. Conf. COMSWARE, 2008, pp. 791-798.

[29] www.i-scoop.eu

[30] D. Locke, "MQ telemetry transport (MQTT) v3. 1 protocol specification," IBM developer Works, Markham, ON, Canada, Tech. Lib., 2010. [Online]. Available: Http://Www.Ibm.Com/Developerworks/ Webservices/Library/Ws-Mqtt/Index.Html

[31] U. Hunkeler, H. L. Truong, and A. Stanford-Clark, "MQTT-SA publish/subscribe protocol for wireless sensor networks," in Proc. 3rd Int. Conf. COMSWARE, 2008, pp. 791-798.

[32] https://www.ibm.com/blogs/internet-of-things/iotapplicationsindustries

[33] M. Darianian, m.p. Michael, smart home mobile RFID-based internet of-things systems, and services, 2008 international conference on advanced computer theory and engineering. (2008) 116120 .

[34] A.M. Vilamovska, E. Hattziandreu, R. Schindler, C. Van Oranje, H. DeVries, J. Krapelse, RFID Application in HealthcareScoping and Identifying Areas for RFID Deployment in Healthcare Delivery, RAND Europe, February 2009

[35] H. Jun-Wei, Y. Shouyi, L. Leibo, Z. Zhen, W. Shaojun, A Crop Monitoring System Based on Wireless Sensor Network, Procedia Environmental Sciences. 11 (2011) 558-565.

[36] www.link-labs.com/blog/iot-agriculture.

[37] R.k. rana, c.t. chou, S.S. kanhere, N. Bulusu, w. Hu, ear-phone: an end to-end participatory urban noise mapping system, in: ACM request permissions, 2010

[38]https://en.wikipedia.org/wiki/Advanced_driverassistance_systems

[39] "Arduino due".

[40] Red wire Econotag II".

[41] "Z1 datasheet".

[42] "IoT-LAB: Very large scale open wireless sensor network testbed".

[43] "OpenMote-CC2538".

[44] "Telos-Ultra Low Power IEEE 802.15.4 compliant wireless sensor module".

[45] C. Bormann, M. Ersue, A. Keranen, "Terminology for constrained node networks", May 2014.

[46] R. Jedermann, T. Pötsch, C. Lloyd, "Communication techniques and challenges for wireless food quality monitoring", Philos. Trans. Roy. Soc. London A: Math. Phys. Eng. Sci., vol. 372, 2017.

[47] W. Dong, C. Chen, X. Liu, J. Bu, "Providing OS support for wireless sensor networks: Challenges and approaches", IEEE Commun. Surv. Tuts., vol. 12, no. 4, pp. 519-530, 2010

[48] L. Saraswat, P. S. Yadav, "A comparative analysis of wireless sensor network operating systems", Int. J. Eng. Technosci., vol. 1, no. 1, pp. 41-47, 2010.

[49] R. Min et al., "Energy-centric enabling technologies for wireless sensor networks", IEEE Wireless Commun., vol. 9, no. 4, pp. 28 39, Aug. 2002

[50] J.-H. Hoepman, B. Jacobs, "Increased security through open source", Commun. ACM, vol. 50, no. 1, pp. 79-83, Jan. 2007

[51] A. Dunkel's, B. Grönvall, T. Voigt, "Contiki-A lightweight and flexible operating system for tiny networked sensors", Proc. 29th Annu. Int. Conf. Local Computer. Network. (LCN), pp. 455-462, 2004.

[52] "Contiki operating system".

[53] P. Levis, W. Weber, J. M. Rabaey, E. Aarts et al., "TinyOS: An operating system for sensor networks" in Ambient Intelligence, Berlin, Germany: SpringerVerlag, pp. 115-148, 2005

[54] E. Baccelli, O. Hahm, M. Günes, M. Wählisch, T. C. Schmidt "RIOT OS: Towards an OS for the Internet of Things", Proc. 32nd IEEE INFOCOM, 2013

[55] "RIOT operating system".

[56] Berkeley's OpenWSN project".

[57] "CNN lite: Lightweight implementation of the content centric networking protocol", 2014.

[58] R. Barry, "Free RTOS a free open source RTOS for small embedded real-time systems".
[59] "Embedded Linux/microcontroller project".

[60] "NuttX real-time operating system-NuttX real-time operating system", 2015

[61] https://rtos.com/solutions/threadx/real-time-operating-system/.

[62] www.windriver.com/products/vxworks/.

[63] https://www.sysgo.com/products/pikeos-hypervisor/why-pikeos/.

[64] https://www.mentor.com/embedded-software/nucleus/".

[65] www.operating-system.org/betriebssystem/_english/bswince.htm.

[66] Alexandrous Plantelopoulous and Nikolaos. G. Bourbakis, "A Survey on Wearable sensor based system for health monitoring and prognosis," IEEE Transaction on system, Man and Cybernetics, Vol.40, No.1, January 2010. 